\title{
Common Sensitive Prognostic Marker in Breast Cancer and their Clinical Significance: A Review Article
}

\author{
Ahmed Fawzy ${ }^{1,2}$, Yaser Abdulsalam Alqelaiti ${ }^{3 *}$, Mohammed Mubrik Almatrafi ${ }^{3}$, Omar Mohammad Almatrafi ${ }^{3}$, Elaaf Abdulsalam \\ Alqelaiti $^{3}$
}

\begin{abstract}
${ }^{1}$ Department of General Surgery, Faculty of Medicine, Taibah University, Al Madinah Al Munawara, Saudi Arabia. ${ }^{2}$ Department of General Surgery, Faculty of Medicine, Menoufia University, Egypt. ${ }^{3}$ College of Medicine, Taibah University, Madinah, Saudi Arabia.
\end{abstract}

\section{Abstract}

The most common cancer in women is breast cancer. Also, it is the biggest cause of death in women with cancer. The exact cause is not known but there are risk factors such as women above 55, Caucasian races, late age of first pregnancy, genetic factors and, family history. It has two classifications histological and molecular. Tumor biomarkers are either prognostic markers or predictive markers. We here present a review for breast cancer prognostic markers. Breast cancer prognostic markers are divided into classical (ER. PR, Ki67, and HER2) and novel (P53, cyclin E, cyclin D1, BRCA 1-2, VEGF, and TBX 2-3). The objective of this review is to evaluate the important prognostic marker for breast cancer and to provide its clinical significance.

The use of biomarkers helps breast cancer patients to get the best treatment. The prognostic markers are to evaluate the outcome. They either indicate for good prognosis as ER and PR or indicate for bad prognosis as P53, HER2, cyclin E, cyclin D1, BRCA1, BRCA2, VEGF, TBX2, and TBX3. Also, some can be used as an important indicator for tumor recurrence as ER, Ki67, P53, and cyclin E. Tumor prognostic markers are clinically important for breast cancer patients. We recommend its clinical use to help breast cancer patients get the best treatment and prognosis options.

Keywords: Breast cancer, Prognostic markers, Diagnosis, Management

\section{INTRODUCTION}

Globally, the most common cancer in females is breast cancer. It affects more than 1.5 million per year and it is the biggest cause of death in women with cancer [1]. In Saudi Arabia, breast cancer is represent $28.7 \%$ of cancer in women in 2014 according to the National Cancer Registry in Saudi Arabia [2]. Also, the percentage of breast cancer in Saudi patients is significantly at an earlier age than in Western countries [3].

The exact causes are not well known but many risk factors are proved. Women above 55 (post-menopause), the Caucasian races, late age of first pregnancy are more expected to have breast cancer. Genetic factors and family history are playing also a big role in breast cancer. Over $10 \%$ of the western countries' breast cancer patients have a genetic factor. Some studies have linked alcohol consumption and breast cancer. Another important risk factor is obesity, especially in postmenopausal breast cancer. Also, some studies proved occupationally related risk factors [4-7].

Breast cancer is histologically classified as mucinous A, mucinous B, invasive lobular carcinoma (ILC), invasive ductal carcinoma with osteoclastic giant cells, apocrine, tubular, neuroendocrine, adenoid cystic, micropapillary, metaplastic, and medullary carcinoma. The molecular classification of breast cancer includes Her2+, basal-like, and luminal. It is important to know the type of cancer because each type has its clinical features and therapeutic strategies $[8,9]$.

The American Joint Committee on Cancer (AJCC) suggestion for breast cancer staging is based on 7 key information (A) size of the tumor (B) spread to nearby lymph nodes (C) metastasis (D) Estrogen Receptor present (E) Progesterone Receptor present (F) Her2/neu (Her2) status amount (G) Grade of the cancer. According to this staging the therapeutic strategies and prognostics are made [10].

It is important to know the patient complains about breast cancer. First and the most important is there a lump and if not,

Address for correspondence: Yaser Abdulsalam Alqelaiti,
College of Medicine, Taibah University, Madinah, Saudi Arabia.
yaser.alqelaiti @ gmail.com

This is an open-access article distributed under the terms of the Creative Commons Attribution-Non Commercial-Share Alike 3.0 License, which allows others to remix, tweak, and build upon the work non commercially, as long as the author is credited and the new creations are licensed under the identical terms.

How to cite this article: Fawzy A, Alqelaiti Y A, Almatrafi M M, Almatrafi O M, Alqelaiti E A. Common Sensitive Prognostic Marker in Breast Cancer and their Clinical Significance: A Review Article. Arch. Pharm. Pract. 2022;13(1):405. https://doi.org/10.51847/T8D3Bp2119 
swelling of the breast. Also, other sign like skin irritation sometimes orange peel. Pain or redness is another sign. The nipple is also important it may be retracted or may be discharge comes from it [11].

Breast cancer is diagnosed by clinical examination and imaging then come the pathological assessment to confirm. In the clinical examination, there is breast palpation and lymph nodes. Mammography and ultrasound are used to image the breast and regional lymph nodes. The pathological assessment is preforming by needle or cut pathological biopsy and it should be taken before any treatment [12].

In the past 20 years, tumor biomarkers have been more understood because of technological advances. Tumor biomarkers divide into two groups which are prognostic markers and predictive markers. The prognostic markers are used to evaluate the outcome and the predictive markers use to evaluate the clinical intervention benefit [13]. This article is mainly about prognostic breast cancer markers that are divided into classical (ER. PR, Ki67, and HER2) and novel (P53, cyclin E, cyclin D1, BRCA 1-2, VEGF, and TBX 2-3) [14].

\section{Objective}

The objective of this review is to evaluate the important prognostic marker for breast cancer and to provide its clinical significance.

\section{Materials and Methods}

Clinical trials as per the WHO definition is a research type that look into new treatment compounds and assesses their effects on human well-being [9, 10]. they are mostly done in five phases with increasingly accurate processes in every phase. Compounds that are discontinued from the phases are found inefficient or harmful. Before the clinical trials, the new drug undergoes pre-clinical studies. These studies are done in vivo (animal populations) and in vitro (laboratory). In vitro substrate or animal, subjects are given different dosages of the study drug to get pharmacokinetic quantities, toxicity, and preliminary efficacy to assist pharmaceutical organizations and researchers in making the decision whether it is advantageous to proceed with further testing [15].

\section{Review of Literature}

\section{Estrogen Receptor (ER)}

ER is a nuclear steroid receptor. Its function is transcription regulator and $17 \mathrm{p}$-estradiol estrogen hormone is responsible for its control. It is playing a role in proliferation, differentiation, and cellular growth. Also, it is having a critical role in breast cancer pathophysiology. ER is known for its key role in the puberty elongation of mammary ductal and lactational differentiation of the lobules. Based on the following of the response to the treatment, ER is the most significant marker [16]. It uses to see the suitability of the patient to have endocrine therapy like raloxifene, and tamoxifen benefit [17]. Clinically, ER-a positive is associated with a good prognosis involving cell proliferation lower rate and tumor differentiation histological evidence [18]. There is the study found that the status of ER-a predicts skeletal metastasis late-onset [19]. Positive ER tumors are at greater risk of relapse than negative ER tumors [20].

The response rate to endocrine therapy is directly proportional to ER content. The clinical outcome for a patient with positive ER/ positive PR tumors is better than negative ER/ negative PR tumors [21]. In Carcinoma In-Situ (CIS) the role of ER has particular attention. Patient with ER-negative has worse outcome in CIS [22].

\section{Progesterone Receptor (PR)}

$\mathrm{PR}$ is a nuclear hormone receptor and it is presenting on chromosome 1 Iq22 as a single gene. It subdivides into PR-A and PR-B and their activity is different [23]. PR target genes produce a protein that has a role in cell growth, apoptosis, transcription, steroid, and lipid metabolism, and some of the proteins are correlated with breast cancer or mammary gland development [16]. In treating breast cancer and endometrial hyperplasia PR has an important therapeutic role [24]. It has a chief position in the lobuloalveolar and ductal growth of the conventional mammary gland.

Analysis of PR is important for every invasive breast cancer and metastasis as well because the result will influence the treatment plan [25]. The tumor size and grade are negatively correlated with high PR levels. Also, PR is used as a prognostic marker in ER-positive breast cancer patients. Studies show that ER-positive expression of PR improves the survival rate of patients using estrogen receptor therapy [14].

\section{Ki67}

Gerdes et al. in 1983 found Ki67 (also known as MKI67): a nuclear non-histone protein that encodes in the MKI67 gene. It appears only in the cycle of the cell proliferative phases (G1, S, G2, and M). Ki67 is important for the proliferation of the cells because, without it, there will be prevention of cell proliferation [26]. The function of Ki67 has been difficult to determine but its role suggests to be in organizing DNA. Also, it suggests that $\mathrm{Ki67}$ is playing a role in ribosomes synthesis in cell division [14].

$\mathrm{Ki} 67$ is a prognostic marker that broadly has been used for the early stage of breast cancer. There is a strong correlation between Ki67 positive cells percentage and age, mitotic rate, and nuclear grade [27]. As indicated by some studies $20 \%$ to half of the Ki67 expression in cells shows a high risk of the disease recurring. During breast cancer endocrine therapy, Ki67 expression was inversely proportional to recurrencefree survival better after a fortnight of adjuvant therapy than before it [14]. In a study done by Petrelli et al., the prognostic value of $\mathrm{Ki}-67$ has an independent in terms of overall survival in breast cancer patients. The greatest analytical significance 
threshold of Ki-67 is yet unknown, but the greater risk of death is associated with a cut-off $>25 \%$ compared with lower expression rates [28]. Ki67 is a laboratory test important and useful in breast cancer patients' prognosis because it is technologically easy and closely linked to clinical results [14].

\section{Human Epidermal Growth Factor Receptor-2 (HER2)}

HER2 (mainly known as HER2/neu) is a protooncogene that belongs to the epidermal growth factor receptor (EGFR) family. In the type I subfamily there are from HER2 to HER4. The HER2 receptor is playing a role in cell differentiation, multiplication, and subsistence. The HER2 gene is in the transmembrane tyrosine kinase receptor protein encode chromosome [29].

Circulating HER2 receptor protein levels use to predict the progression and presence of positive HER 2 cells. Also, its protein level is used as a prognostic marker that indicates survival as ER and PR expression or tumor size [29]. It founds that the overexpression of HER2 receptor protein is correlating to a significant decrease in survival rate for the breast cancer patient. Also, it reported that the appearance of HER2 receptor protein is useful in relapsing early diagnosis and the envisioning of what will happen to metastases of breast cancer [30].

HER2 expression is higher than normal in $30 \%$ of patients with breast cancer and the main cause of its overexpression is gene intensification. From these cases, ductal carcinomas in situ are in $60 \%$ of patients, and infiltrating breast carcinomas are in 20\%-30\% of patients. overexpression of the HER2 in alar lymph node-positive breast cancer patients is correlated with poor disease-free survival (DFS) rate. Overexpression of HER2 is significantly worse overall survival rate there is twice the mortality rate in these patients. High HER2 is more present in high nuclear grade tumors $29 \%$ than low nuclear grade tumors $12 \%$ [14].

In some studies, the patients have breast cancer metastatic with overexpression of HER2 use chemotherapy and trastuzumab is correlate with slow progression of the disease, a higher rate and longer time of response, death at 1 year was lower, longer survival, and risk of death decreased. HER2 overexpression patients have response rates from $67 \%$ to $81 \%$ while HER2 normal expression patients have $41 \%$ to $46 \%$ response rates. These response rates in a patient with different expressions of HER2 are significant statistically in all test techniques, with TAB250 and CB11 antibodies, and the powerful implication is in fluorescent in situ hybridization (FISH).

\section{P53}

The cell cycle regulation by tumor suppression is a role played by P53 is a nuclear protein. This has contributed to cancer prevention and Its gene is located on chromosome
$17 \mathrm{p} 13$ [31]. P53 is the most popular mutation of genes identified in human tumors. Breast cancer with P53 mutation is correlated with worse overall survival and more aggressive disease. The appearance of the mutation is early in cancer progression and is approximately appears in $22 \%$ of fatal breast tumors [16].

P53 status is used in the prediction of response to chemotherapy. Death of the tumor cell by apoptosis after exposure to radiotherapy or chemotherapy depends on P53, so the radiotherapy and chemotherapy induce damage to the DNA with detect damage to P53 is unable to repair and triggers apoptosis. Thus, it suggests that P53 reduced function tumor is resistant to chemotherapy and radiotherapy [32].

P53 mutations are confirmed to have an overall bad and disease-free survival in the breast cancer patient. In several studies, the most forthcoming indicator of persistence or morbidity is P53. Studies have shown that P53 mutation has a strong prognostic significance in breast cancer [14].

\section{Cyclin E}

Cyclin E is a 50-kd cell cycle regulator protein. It is shown in the cell cycle final phase. Cell cycle regulatory proteins disturbance is playing an important role in cancer. Cyclin $\mathrm{E}$ and cyclin-dependent kinase-2 (CDK2) is forming an active complex to allow the development through the G1 phase of the cell cycle and control of entering the $\mathrm{S}$ phase. Cyclin $\mathrm{E}-$ CDK2 complex enzyme activity is inhibited by the p21 and p27 proteins. The disparity of the levels of CDKs inhibitors, cyclins, and CDKs causes the hysterical division of cells [33]. In about $25 \%$ of breast tumors, the cyclin E is extremely high or unusually constant as contrasted to normal cells. Overexpression of cyclin $\mathrm{E}$ is motivating variances in gene expression sequences that correlate with the adhesion of cells in addition to a lowered capability of movement and invasion of functional assays. High cyclin E protein level is correlated with meager breast cancer prognosis. Moreover, the risk of recurrence of breast cancer is increased with overexpression of cyclin E [34].

\section{Cyclin D1}

Cyclin D1 is a G1 phase progression key regulator protein. In response to growth factors cyclin D1 protein is synthesized and in the middle of the G1 phase of the cell cycle reach its peak level. it is excreted by the CCND1 gene located on chromosome 11q13. In breast cancer, cyclin D1 gene Amplification is detected a number of cases [14].

There are both negative and positive findings reported concerning the excess cyclin D1. In ER-positive patients, the relationship between poor disease outcome and cyclin D1 gene amplification comes out. However, other studies show good prognosis correlates with cyclin D1 protein expression. Also, other studies did not confirm this relationship [14]. 
For special circumstances, it is more complicated. Overexpression of cyclin D1 can make the clinical outcome worse by giving endocrine treatment resistance. These issues ought to contain more cyclin D1 analysis excretion and patient results to the found decision [14].

\section{BRCA1 and BRCA2}

BRCA1 and BRCA2 (BReast CAncer gene) are growth withholding genes. Their locations are $13 \mathrm{q}$ and $17 \mathrm{q}$ respectively according to a 20th -century discovery. Their genes encode cell growth inhibit factors and these factors also participate in gene transcription regulation, cell cycle control, apoptosis, DNA damage repair, and other important processes in the cell [35]. The most common mutations in BRCA1 are 185 del AG, 5382 ins C, 4153 del A, and 3819 del 5, and the most common mutations in BRCA2 are 5802 del4 and 4075 del GT [36]. The mutation in BRCA1 and BRCA2 genes are associated with an increase in lifetime risk for several malignant tumors, particularly breast cancer and ovarian cancer [35].

There are only $5 \%$ to $10 \%$ inherited breast cancer cases. By the age of 70 , there are $55 \%$ to $65 \%$ carriers of BRCA1 metamorphosis, and $45 \%$ carriers of BRCA2 mutation will get breast cancer [37]. A recent study in 21401 families suspected to have a mutation in the BRCA gene shows that BRCA1 or BRCA2 mutation is in $24 \%$ of these families [38]. The effect of BRCA mutations is controversially correlated with poor prognosis. There is a study showing that the 10year survival rate is the same for either the patients with or without the BRCA1 mutation [39]. Another study shows that breast cancer patients with a BRCA2 mutation are less likely to survive than the ones without it. However, the predictions are tightly contained to the carriers with diploid, tumors that are slowly multiplying [40]. Templeton et al. comprising data from 10,180 patients from 16 studies and concluded that mutations in BRCA were not correlated with worse overall survival [41].

\section{Vascular Endothelial Growth Factor (VEGF)}

Vascular endothelial growth factor (VEGF) is a family that includes five polypeptide growth elements (VEGF A, VEGF $\mathrm{B}$, VEGF C, VEGF $\mathrm{D}$, and placenta-like growth factor (PLGF)). VEGF is significant because of its lymphangiogenic and angiogenic qualities that enhance the development and metastasis of neoplasms. VEGF-C is a responsible factor for the regulation of lymph-angiogenesis. Overexpression of VEGF-C is present in lung, gastric, colon, prostatic, and breast cancers, and it is correlated with lymph node metastasis, and prognosis [42].

VEGF and angiogenesis are critical for metastasis and tumor growth. In general, in human breast cancer tissue, VEGF was one of the most important tumor angiogenesis mediators and poor survival associate with elevated VEGF levels. Spreading of cancer cells from the initial location to further sites is the most important factor of breast cancer patients' survival [14]. Shivakumar et al. show that overexpression of VEGF was found in metastatic patients compared to benign lesions and was correlated positively with tumor grade [43].

\section{$T B X 2$ and $T B X 3$}

TBX2 and TBX3 are T-box proteins that have a T-domain which has effects on dimerization and DNA combination. TBX2 is from the Tbx subclass of T-box transcription elements. In mammals, the only T-box factors with reported suppressor functions are TBX2 and TBX3. TBX2 is important for the various tissues and organs morphogenesis like heart, bone, limbs, and mammary glands. The evidence link between TBX2 and tumorigenesis. TBX2 and TBX3 downregulation of the ARF growth suppressor which is implicated in tumor development. Expression of TBX2 upregulated in several types of cancer such as melanoma, breast cancer, lung cancer, pancreatic cancer, colorectal cancer, and bladder cancer [44].

TBX2 is reported to increase in $8.6 \%$ to $21.6 \%$ of sporadic human breast carcinomas where overexpress the protein. TBX2 engages in the carcinogenesis of breast cancer by accelerating cell proliferation, and DNA ploidy. Also, the TBX2 overexpression is making cells resistant to chemotherapy [14].

Table 1 shows a summary of these prognostic markers and their significance (annexes page 18).

Table 1. Summary for the important marker and its significant

\begin{tabular}{cc}
\hline Marker & Significant \\
\hline ER & $\begin{array}{c}\text { ER-a positive is associated with a good prognosis involve } \\
\text { cell proliferation lower rate and tumor differentiation } \\
\text { histological evidence. Positive ER tumors are at greater risk } \\
\text { of relapse than negative ER tumors. }\end{array}$
\end{tabular}

The tumor size and grade are negatively correlated with high PR levels. PR is used as a prognostic marker in ER-

PR positive breast cancer patients. In ER-positive expression of PR improve the survival rate of the patients using estrogen receptor therapy.

Ki67 is broadly used for the early stage of breast cancer. There is a strong correlation between Ki67 positive cells Ki67 percentage and age, mitotic rate, and nuclear grade. Ki67 express in more than $20 \%-50 \%$ of the cells means that the risk of recurrent disease developing in breast cancer is high.

Overexpression of HER2 receptor protein is correlating to a significant decrease in survival rate for breast cancer in relapsing early diagnosis and in predicting the fate of metastases of breast cancer. Overexpression of HER2 is significantly worse overall survival rate there is twice the mortality rate in these patients.

P53
P53 mutation is the most powerful recurrence and morbidity marker. 
Fawzy et al.: Common Sensitive Prognostic Marker in Breast Cancer and their Clinical Significance: A Review Article

$\begin{array}{cc}\text { Cyclin } & \text { High cyclin E protein level is correlated with poor } \\ \text { E } & \text { predictions in breast cancer. Also, the risk of recurrence of } \\ \text { breast cancer is increased with overexpression of cyclin E. } \\ \text { Cyclin D1 overexpression and the outcome have been } \\ \text { controversial, there are both positive and negative findings } \\ \text { reported. Overexpression of cyclin D1 can make the } \\ \text { clinical outcome worse by giving endocrine treatment } \\ \text { resistance. }\end{array}$

\section{CONCLUSION}

Tumor prognostic markers are clinically important for breast cancer patients. We recommend its clinical use to help breast cancer patients get the best treatment. It shows either indicate for good prognosis as ER and PR or indicate for bad prognosis as P53, HER2, cyclin E, cyclin D1, BRCA1, BRCA2, VEGF, TBX2, and TBX3. Also, it is important in the indication of recurrent tumor as ER, Ki67, P53, and cyclin E.

\section{ACKNOWLEDGMENTS: None CONFLICT OF INTEREST: None FINANCIAL SUPPORT: None ETHICS STATEMENT: None}

\section{REFERENCES}

1. World health organization WHO [internet]. [cited 2018 Feb 10]. Available from: http://www.who.int/cancer/prevention/diagnosisscreening/breast-cancer/en/

2. Al-Shahrani Z, Al-Rawaji A, Al-Madouj AN, Hayder MS, Al-Zahrani A, Al-Mutlaq H, et al. Cancer Incidence Report Saudi Arabia 2014. Saudi Cancer Regist. 2017:1-81. Available from: http://www.chs.gov.sa/ar/HealthCenters/NCC/CancerRegistry/Cancer RegistryReports/2014.pdf

3. Saggu S, Rehman H, Abbas ZK, Ansari AA. Recent incidence and descriptive epidemiological survey of breast cancer in Saudi Arabia. Saudi Med J. 2015;36(10):1176-80.

4. John Hopkins medicine [Internet]. [cited $2018 \mathrm{Feb} 10]$. Available from: https://www.hopkinsmedicine.org/healthlibrary/conditions/adult/breas t_health/risk_factors_for_breast_cancer_85,P00160

5. McPhearson K, Steel C, Dixon J. Breast cancer-epidemiology, risk factors and genetics. Brit Med J. 2000;321(7261):624-8.

6. Morimoto LM, White E, Chen Z, Chlebowski RT, Hays J, Kuller L, et al. Obesity, body size, and risk of postmenopausal breast cancer: the Women's Health Initiative (United States). Cancer Causes Control. 2002;13(8):741-51.

7. Rafnsson V, Tulinius H, Jónasson JG, Hrafnkelsson J. Risk of breast cancer in female flight attendants: a population-based study (Iceland). Cancer Causes Control. 2001;12(2):95-101.

8. Weigelt B, Horlings HM, Kreike B, Hayes MM, Hauptmann M, Wessels LF, et al. Refinement of breast cancer classification by molecular characterization of histological special types. J Pathol. 2008;216(2):141-50.

9. Dai X, Li T, Bai Z, Yang Y, Liu X, Zhan J, et al. Breast cancer intrinsic subtype classification, clinical use and future trends. Am J Cancer Res. 2015;5(10):2929-43.
10. American Cancer Society [Internet]. [update 2017 December 20; cited $2018 \mathrm{Feb}$ 10]. Available from: https://www.cancer.org/cancer/breastcancer/understanding-a-breast-cancer-diagnosis/stages-of-breastcancer.html

11. American Cancer Society [Internet]. [update 2017 September 22; cited $2018 \mathrm{Feb} 10]$. Available from: https://www.cancer.org/cancer/breastcancer/about/breast-cancer-signs-and-symptoms.html

12. Senkus E, Kyriakides S, Ohno S, Penault-Llorca F, Poortmans P, Rutgers E, et al. Primary breast cancer: ESMO Clinical Practice Guidelines for diagnosis, treatment and follow-up. Ann Oncol. 2015;26(5):v8-v30.

13. Mehta S, Shelling A, Muthukaruppan A, Lasham A, Blenkiron C, Laking G, et al. Predictive and prognostic molecular markers for cancer medicine. Ther Adv Med Oncol. 2010;2(2):125-48.

14. Taneja P, Maglic D, Kai F, Zhu S, Kendig RD, Elizabeth AF, et al. Classical and novel prognostic markers for breast cancer and their clinical significance. Clin Med Insights Oncol. 2010;4:15-34.

15. Kurdi L, Alhusayni F. Cytotoxicity effect of 5-fluorouracil and bee products on the MCF-7 Human Breast Cancer Cell Line in vitro. Int J Pharm Phytopharmacol Res. 2020;10(2):19-26.

16. Kabel AM. Tumor markers of breast cancer: New prospectives. J Oncol Sci. 2017;3(1):5-11.

17. Kabel AM, Elkhoely AA. Ameliorative potential of fluoxetine/raloxifene combination on experimentally induced breast cancer. Tissue Cell. 2016;48(2):89-95.

18. Lumachi F, Brunello A, Maruzzo M, Basso U, Mm Basso S. Treatment of estrogen receptor-positive breast cancer. Curr Med Chem. 2013;20(5):596-604.

19. Han HH, Lee SH, Kim BG, Lee JH, Kang S, Cho NH. Estrogen receptor status predicts late-onset skeletal recurrence in breast cancer patients. Medicine. 2016;95(8).

20. Duffy MJ, Harbeck N, Nap M, Molina R, Nicolini A, Senkus E, et al. Clinical use of biomarkers in breast cancer: Updated guidelines from the European Group on Tumor Markers (EGTM). Eur J Cancer. 2017;75:284-98.

21. Bae SY, Kim S, Lee JH, Lee HC, Lee SK, Kil WH, et al. Poor prognosis of single hormone receptor-positive breast cancer: similar outcome as triple-negative breast cancer. BMC Cancer. 2015;15(1):138.

22. Chan M, Chang MC, González R, Lategan B, del Barco E, VeraBadillo F, et al. Outcomes of estrogen receptor negative and progesterone receptor positive breast cancer. PloS one. 2015;10(7):e0132449.

23. Jacobsen BM, Horwitz KB. Progesterone receptors, their isoforms and progesterone regulated transcription. Mol Cell Endocrinol. 2012;357(1-2): 18-29.

24. Giulianelli S, Molinolo A, Lanari C. Targeting progesterone receptors in breast cancer. Vitam Horm. 2013;93:161-84.

25. Lanari C, Wargon V, Rojas P, Molinolo AA. Antiprogestins in breast cancer treatment: are we ready? Endocr Relat Cancer. 2012;19(3):R35-50.

26. Brown DC, Gatter KC. Ki67 protein: the immaculate deception?. Histopathology. 2002;40(1):2-11.

27. Mohamed AA, Obaid NE, Abdelghani S, Alfahed A, Waggiallah HA, Eltayeb LB. Immunohistochemical expression of survivin and KI-67 as tumor markers in breast cancer infected females: A cross-sectional study. Pharmacophore. 2020;10(5):41-5.

28. Petrelli F, Viale G, Cabiddu M, Barni S. Prognostic value of different cut-off levels of Ki-67 in breast cancer: a systematic review and metaanalysis of 64,196 patients. Breast Cancer Res Treat. 2015;153(3):47791.

29. Krishnamurti U, Silverman JF. HER2 in breast cancer: a review and update. Adv Anat Pathol. 2014;21(2):100-7.

30. Rimawi MF, Schiff R, Osborne CK. Targeting HER2 for the treatment of breast cancer. Annu Rev Med. 2015;66:111-28.

31. Kabel AM. Tumor protein p53: Novel aspects of an old tumor marker. J Cancer Res Ther. 2015;3(2):25-7.

32. Parrales A, Iwakuma T. Targeting oncogenic mutant p53 for cancer therapy. Front Oncol. 2015;5:288.

33. Trovesi C, Manfrini N, Falcettoni M, Longhese MP. Regulation of the DNA damage response by cyclin-dependent kinases. J Mol Biol. 2013;425(23):4756-66. 
34. Bi H, Li S, Qu X, Wang M, Bai X, Xu Z, et al. DEC1 regulates breast cancer cell proliferation by stabilizing cyclin E protein and delays the progression of cell cycle S phase. Cell Death Dis. 2015;6(9):e1891.

35. Zhu Y, Wu J, Zhang C, Sun S, Zhang J, Liu W, et al. BRCA mutations and survival in breast cancer: an updated systematic review and metaanalysis. Oncotarget. 2016;7(43):70113-27.

36. Wang F, Fang Q, Ge Z, Yu N, Xu S, Fan X. Common BRCA1 and BRCA2 mutations in breast cancer families: a meta-analysis from systematic review. Mol Biol Rep. 2012;39(3):2109-18.

37. Godet I, Gilkes DM. BRCA1 and BRCA2 mutations and treatment strategies for breast cancer. Integr Cancer Sci Ther. 2017;4(1).

38. Kast K, Rhiem K, Wappenschmidt B, Hahnen E, Hauke J, Bluemcke $\mathrm{B}$, et al. Prevalence of BRCA1/2 germline mutations in 21401 families with breast and ovarian cancer. J Med Genet. 2016;53(7):465-71.

39. Huzarski T, Byrski T, Gronwald J, Górski B, Domagała P, Cybulski C, et al. Ten-year survival in patients with BRCA1-negative and BRCA1positive breast cancer. J Clin Oncol. 2013;31(26):3191-6.

40. Tryggvadottir L, Olafsdottir EJ, Olafsdottir GH, Sigurdsson H, Johannsson OT, Bjorgvinsson E, et al. Tumour diploidy and survival in breast cancer patients with BRCA2 mutations. Breast Cancer Res Treat. 2013;140(2):375-84.

41. Templeton AJ, Gonzalez LD, Vera-Badillo FE, Tibau A, Goldstein R, Seruga B, et al. Interaction between hormonal receptor status, age and survival in patients with BRCA1/2 germline mutations: a systematic review and meta-regression. PloS One. 2016;11(5):e0154789.

42. Cheng D, Liang B, Li Y. Serum vascular endothelial growth factor (VEGF-C) as a diagnostic and prognostic marker in patients with ovarian cancer. PLoS One. 2013;8(2):e55309.

43. Shivakumar S, Prabhakar BT, Jayashree K, Rajan MG, Salimath BP. Evaluation of serum vascular endothelial growth factor (VEGF) and microvessel density (MVD) as prognostic indicators in carcinoma breast. J Cancer Res Clin Oncol. 2009;135(4):627-36.

44. Lv Y, Si M, Chen N, Li Y, Ma X, Yang H, et al. TBX2 over-expression promotes nasopharyngeal cancer cell proliferation and invasion. Oncotarget. 2017;8(32):52699-707. 